This is a self-archived version of an original article. This version may differ from the original in pagination and typographic details.

Author(s): Selenius, Elli; Malola, Sami; Kuisma, Mikael; Häkkinen, Hannu

Title: Charge Transfer Plasmons in Dimeric Electron Clusters

Year: 2020

Version: Accepted version (Final draft)

Copyright: @ 2020 American Chemical Society

Rights: In Copyright

Rights url: http://rightsstatements.org/page/lnC/1.0/?language=en

Please cite the original version:

Selenius, E., Malola, S., Kuisma, M., \& Häkkinen, H. (2020). Charge Transfer Plasmons in Dimeric Electron Clusters. Journal of Physical Chemistry C, 124(23), 12645-12654.

https://doi.org/10.1021/acs.jpcc.0c02889 


\title{
Article
}

\section{Charge Transfer Plasmons in Dimeric Electron Clusters}

\author{
Elli Selenius, Sami Malola, Mikael Kuisma, and Hannu Häkkinen
}

J. Phys. Chem. C, Just Accepted Manuscript • DOI: 10.1021/acs.jpcc.0c02889 • Publication Date (Web): 18 May 2020

Downloaded from pubs.acs.org on May 19, 2020

\section{Just Accepted}

"Just Accepted" manuscripts have been peer-reviewed and accepted for publication. They are posted online prior to technical editing, formatting for publication and author proofing. The American Chemical Society provides "Just Accepted" as a service to the research community to expedite the dissemination of scientific material as soon as possible after acceptance. "Just Accepted" manuscripts appear in full in PDF format accompanied by an HTML abstract. "Just Accepted" manuscripts have been fully peer reviewed, but should not be considered the official version of record. They are citable by the Digital Object Identifier (DOI®). "Just Accepted" is an optional service offered to authors. Therefore, the "Just Accepted" Web site may not include all articles that will be published in the journal. After a manuscript is technically edited and formatted, it will be removed from the "Just Accepted" Web site and published as an ASAP article. Note that technical editing may introduce minor changes to the manuscript text and/or graphics which could affect content, and all legal disclaimers and ethical guidelines that apply to the journal pertain. ACS cannot be held responsible for errors or consequences arising from the use of information contained in these "Just Accepted" manuscripts. 


\title{
Charge Transfer Plasmons in Dimeric Electron Clusters
}

\author{
Elli Selenius, ${ }^{\dagger}$ Sami Malola, ${ }^{\dagger}$ Mikael Kuisma, ${ }^{\ddagger}$ and Hannu Häkkinen*,†, \\ $\dagger$ Department of Physics, Nanoscience Center, University of Jyväskylä, FI-40014 Jyväskylä, \\ Finland \\ $\ddagger$ Department of Chemistry, Nanoscience Center, University of Jyväskylä, FI-40014 \\ Jyväskylä, Finland \\ E-mail: hannu.j.hakkinen@jyu.fi
}




\begin{abstract}
The tunability of the optical response of dimers of metal clusters and nanoparticles makes them ideal for many applications from sensing and imaging to inducing chemical reactions. We have studied charge transfer plasmons in separate and linked dimers of closed-shell electron clusters of 8 and 138 electrons using time-dependent density functional theory. The simple model clusters enable the systematic study of the charge transfer phenomenon from the electronic perspective. To identify the charge transfer plasmons, we have developed an index, the Charge Transfer Ratio, for quantifying the charge transfer nature of the excitations. In addition, we analyze the induced transition density and the electron transitions contributing to the dipole moment at the charge transfer plasmon energies. Our results show that the optical response of the dimers is very sensitive to changes in the inter-cluster separation and in the width of the linking channel, with charge transfer plasmon peaks appearing at low energies for dimers with linking or sufficient electron cloud overlap.
\end{abstract}

\title{
Introduction
}

The optical response of metal clusters and nanoparticles is highly dependent on the shape, size, and dielectric environment of the cluster. ${ }^{1}$ The response of two adjacent particles depends also on the separation. ${ }^{2}$ This tunability makes clusters and cluster assemblies or arrays ideal for many applications, such as surface-enhanced Raman spectroscopy for detecting single molecules, ${ }^{3}$ the molecular ruler for measuring distances in the nanoscale, ${ }^{4}$ cancer imaging and treament, ${ }^{5}$ and driving chemical reactions. ${ }^{6}$

Plasmons are collective excitations of the conductance electrons, where the electron density of the cluster oscillates in resonance with the external electromagnetic field. In plasmonic metal clusters, the main excitation is the localized surface plasmon resonance (LSPR), where the electron density oscillations happen mostly on the surface of the cluster. ${ }^{7}$ There have been several approaches to understand and classify different optical excitations in metal 

The distribution of the induced density ${ }^{8,9}$ and collectivity of the excitation ${ }^{10,11}$ have both been used as criteria for plasmonic resonances. Different plasmonicity indeces based on the strength of the induced potential have also been developed. ${ }^{12,13}$ Recently, Gieseking et al. ${ }^{14}$ proposed a method to identify plasmonic resonances based on three criteria - the collectivity of the excitations, the superatomic character of the excitations, and the additivity of the contributions from the single-particle excitations.

In dimers, the plasmons of the two clusters can couple capacitively to form a bonding dipolar plasmon (BDP). ${ }^{15}$ The energy of the excitation is lowered, causing the BDP peak to be redshifted in comparison to the LSPR peak of the single clusters. ${ }^{2,15}$ For short separations and chemically linked dimers there can also be charge transfer plasmons (CTPs). ${ }^{16,17}$ In these CTPs, the electron density oscillates between the two clusters, making one cluster momentarily positively and the other negatively charged. The CTPs happen at energies lower than BDPs, and thus add a new energy range for the applications of plasmons. Depending on the chemical coupling between the clusters, the CTPs can be caused by electrons tunneling or flowing between the clusters. ${ }^{17}$

Charge transfer plasmons have been experimentally observed in many dimeric nanostructures, such as nanogaps between two gold coated atomic force microscope tips, ${ }^{18}$ gold nanorods connected with a gold nanojunction, ${ }^{19}$ and dimers of various nanoparticles, such as gold nanoshells, ${ }^{16}$ silver spheres, ${ }^{20}$ and gold nanoblocks. ${ }^{21}$ For atomistically precise thiolstabilized (SR) gold nanoclusters, Lahtinen et al. observed an absoprtion peak that they assigned to a tunneling $\mathrm{CTP}$ in covalently linked $\mathrm{Au}_{\sim 250}(\mathrm{SR})_{\mathrm{n}}$ dimers. $^{22}$

Large nanoparticles and dimers with a separation of several nanometers can be treated with classical methods solving the Maxwell equations, and good agreement with experiments have been found for nanoparticle dimers in such cases. ${ }^{23}$ However, going to sizes of a few nanometers ${ }^{24}$ or to sub-nanometer separations, ${ }^{17,25}$ the quantum effects cannot be ignored. Thus, to model dimer systems with small, atom-precise clusters with more accuracy, time- 
dependent density functional theory (TDDFT) has been employed in multiple studies. ${ }^{26-31}$

The drawback of TDDFT is that it is computationally heavy. Therefore, in the region where quantum mechanical effects are important, but the size of the system makes atomistic TDDFT calculations unfeasible, some simplifications have to be made. Xiang et al. have employed the time-dependent orbital-free density functional theory (TD-OFDFT) to study dimers and trimers of sodium clusters consisting of 55 or 1415 atoms in order to reduce the computational cost. ${ }^{32}$ The quantum-corrected model (QCM) ${ }^{33,34}$ includes quantum effects in the optical response of nanoparticles with a small gap by modeling electron tunneling by a fictitious conductive material with the local permittivity reflecting the tunneling probability.

One approach is to combine TDDFT with the simple jellium model to represent the cluster dimers. ${ }^{17,35-38}$ There, the nuclei and core electrons are represented as a uniform, positive background charge, and only the valence electrons are modeled explicitely. For elements where the d-electron screening plays a role, such as gold and silver, a dielectric background can be added to model this effect. The simple jellium model with a constant background density is most suitable for alkali metals, such as sodium, for which the s-type valence electrons are strongly delocalized. Different elements of this group can be represented by tuning the background density to correspond to the experimental density.

Since the jellium model allows for electron tunneling and the spilling of the conductance electrons, it enables charge transfer excitations for small gap sizes, and can thus be used to understand the principles of charge transfer plasmons in dimeric structures. To accurately reproduce experimental results quantitatively, the background charge distribution at the edge of the cluster should be adjusted. In close distances, the atomic structure has been shown to affect the electric field enhancement and the charge transfer current. ${ }^{24,39,40}$ Zhang et al. ${ }^{39}$ have shown that for spherical jellium clusters of about 300 atoms, the jellium model gives the same trend for the BDP as atomistic models using bcc and icosahedral packing. The CTP peak is also reproduced by the jellium model, but only at about a separation of $10 \AA$, whereas for the two atomic arrangements a charge transfer mode was seen already at separations of 
30-40 $\AA$. The results indicate that the main trends in the spectra are reproduced by the jellium model, and at sufficiently small separations, the jellium model is able to describe the CTP.

Here, we have employed the jellium model with TDDFT for dimers of spherical clusters to study charge transfer plasmons from the electronic perspective. The simplicity of the background charge potential eases the investigation of the electronic phenomena, since the ground state electron density has a very simple structure, and the changes in electron density during excitations are therefore easier to distinguish and analyze as compared to atomic structures. Since we are using the same model for all the systems, we can follow the trends in the shape of the spectra and the peak energies even if the jellium model causes a shift compared to atomic clusters.

We studied homo- and heterodimers of two 8-electron or 138-electron clusters and varied the inter-cluster separation between the clusters. We also constructed some linked dimers by connecting the clusters with a cylindrical tunnel of the same jellium background density to gain understanding on the effect of conductive linking to the excitations. The conductive jellium linker establishes a coupling of metallic nature between the clusters, allowing electrons to flow between the clusters during a CTP excitation. These linked clusters serve as simple models for clusters that are connected by a conductive linker, such as one or more conductive ligands or a chain of metal atoms. It is possible to modify the background density of the linker to study the effect of the linker conductance. However, here we use the same density as for the clusters to have a linker with the same metallic character. For atomisic clusters, this kind of linking has been studied by Rossi et al. ${ }^{29}$ who studied CTPs in a system with a narrow atomic contact between to metal clusters.

Following the methods of our previous study of the birth of LSPR in jellium clusters, ${ }^{41}$ we use the induced density and analysis of the contributions of the single-particle excitations to study the nature of the excitations in the dimer systems. In addition, we have developed an index to quantify the charge transfer nature of the excitations, enabling us to recognize 
the excitations with charge transfer character from the spectra and follow the development of the CTPs as the separation gets smaller.

\section{Theory and methods}

The GPAW ${ }^{42,43}$ program, which performs the calculations using a uniform real-space grid, was used for the density functional theory (DFT) calculations. The local density approximation (LDA) after Perdew and Wang ${ }^{44}$ was employed for the exchange-correlation functional. Typically, LDA is considered to yield inappropriate excitation energies due to lack of divergence of exchange-correlation kernel at charge transfer excitations. In the supplementary information (section 1), we discuss why charge transfer plasmons differ from this picture, and why already LDA yields a balanced description of the intra and inter particle Coulomb forces. The optical absorption spectra were calculated with linear response time dependent DFT (lr-TDDFT) as implemented in GPAW. ${ }^{45}$ Some of the peaks in the spectra were then further analyzed using time-dependent density functional perturbation theory (TD-DFPT), ${ }^{46}$ which allows us the solve the contributions of the Kohn-Sham (K-S) electron-hole (e-h) transitions contributing to the excitation and to calculate the induced transition density. The K-S contributions were visualized using the dipole transition contribution map (DTCM) scheme, ${ }^{47,48}$ where the relative strengths of the contributions to the transition dipole moment from all the K-S transitions can be seen at the same time. To study the excitations with charge transfer between the two clusters, the DTCM analysis is done for light polarized parallel to the dimer axis for all the systems.

Since the clusters of the dimers are spherical, the symmetries of the superatom-like electron orbitals were identified by doing the $\mathrm{Y}_{\operatorname{lm}}$ analysis, ${ }^{49}$ where the part of the wavefunction inside a certain projection sphere is projected into spherical harmonics. This analysis was done separately for the two clusters in one dimer, summing the contributions for the projected density of states (PDOS) figures. Thus these symmetries are the symmetries for one 
cluster, not the whole dimer system, where the orbitals are bonding and antibonding states of the single-cluster orbitals. Introducing the conductive pathway breaks the local spherical symmetry already at ground state level and thus allows the charge transfer excitations (also discussed in supplementary section 1).

In the simple jellium model used here, the edges of the positive uniform charge distribution were sharp. The jellium background of a linked dimer can be seen in figure 1 a). The radius of the clusters $r$, the separation between the jellium egdes $S$, and the radius of the cylindrical linker $a$ are shown. The electron density experiences spill-out from the positive core, and this spill-out is relatively larger for smaller clusters. The electron densities of the studied systems along the dimer axis can be seen in figure S1.

For the separate spheres, the density used for the positive background was approximately the density of sodium, calculated from the Wigner-Seitz radius $r_{w s}=2.08 \AA$. For the linked spheres, the volume of the spheres was kept the same, so the volume of the dimer was changed by the volume of the linker. Thus the jellium density is a little smaller for the linked systems, maximum $5.6 \%$ for the 8-electron sphere system with the wider linker.

Because of the smooth jellium background, the grid spacing could be as big as $0.4 \AA$ for the dimers of 8 -electron clusters and $0.5 \AA$ for the dimers of 138 -electron clusters. The convergence of the energies relative to the grid spacing was tested. The energy of the systems was converged with the accuracy of $0.5 \mathrm{meV}$ per electron. In the optical spectrum calculations, the energy cut-off was $5 \mathrm{eV}$ or larger, meaning that at least all the e-h transitions with a smaller energy difference than this were included. All the spectra are plotted for light polarized along the dimer axis.

To analyze the CT nature of the excitations, we introduce the Charge Transfer Ratio (CTR) which is defined in equation 1 , and which can be calculated for any energy in the spectrum:

$$
\mathrm{CTR}=\left|\frac{D \int_{A} \rho_{\text {ind }}(\mathbf{r}) d \mathbf{r}}{\int_{A+B} x \rho_{\text {ind }}(\mathbf{r}) d \mathbf{r}}\right|
$$

Here $\rho_{\text {ind }}$ is the induced transition density at the studied energy, $x$ the coordinate on the 

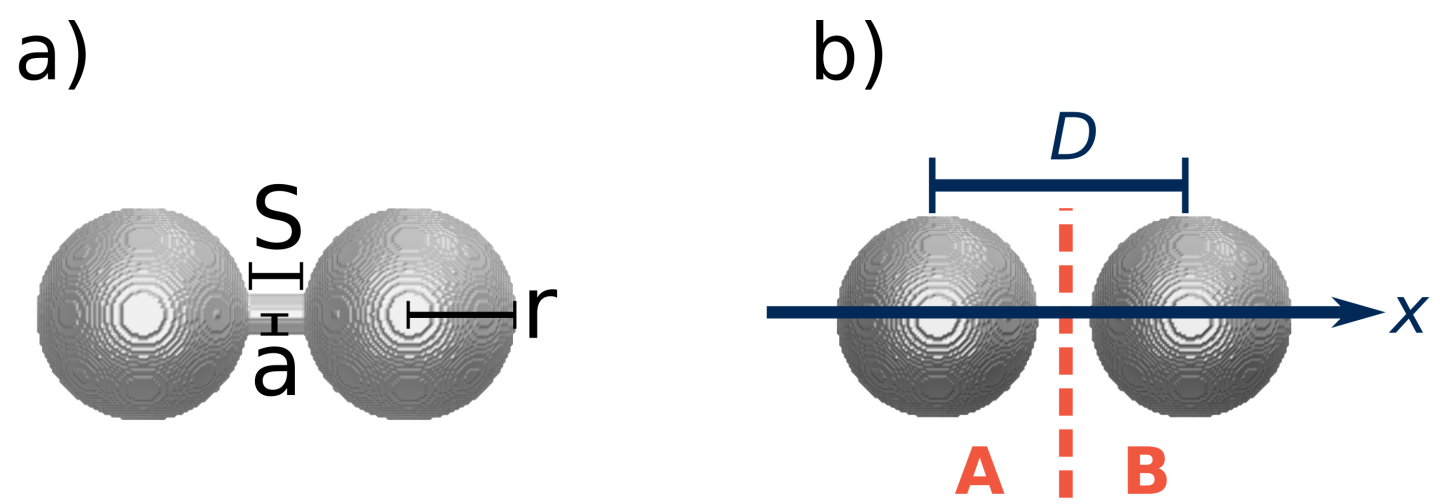

Figure 1: a) A coupled dimer with a radius of the spheres $r$, separation $S$, and radius of the linking channel $a$. Shown is the positive jellium background. b) Explanation of the symbols used in equation 1. $A$ and $B$ are the left and right sides of the calculation box, $D$ is the distance between the centers of the clusters, and $x$ is the direction of the dimer axis.

dipole axis and $D$ the distance between the centers of the two clusters. The calculation box is divided into two equal sides, $A$ and $B$, where the division is in the middle of the gap between the clusters. See figure $1 \mathrm{~b}$ ) for a visual guide to the variables.

The denominator of equation 1 is the dipole moment of the system along the dimer axis, and the numerator is the dipole moment of a system where all the induced charge on side A is concentrated in the center of cluster A, and similarly for side B. The numerator is thus a measure of the charge separation between sides $A$ and $B$, and as a result also between the two clusters. The numerator acts as normalization. The CTR compares the 'CT moment', the expression in the numerator, to the dipole moment: the higher the number is, the bigger the CT nature of the excitation is. If both clusters are neutral, i.e. the electron density oscillates separately inside each cluster, the integral of the induced density on side $A$, and thus the CTR value, is zero. Depending on the distribution of the charges, the ratio can also be negative or greater than one, but the absolute value is the measure of the charge transfer characteristic. With these numbers we can then plot a charge transfer spectrum showing how the charge transfer characteristics of the excitations evolve as a function of energy. Further discussion on the details of the CTR is given in the SI text. 


\section{Results and discussion}

The studied dimer systems are named 1-12. The parameters for each system are shown in table 1 . The separations are given in terms of the radii of the systems, $r \approx 4.2 \AA$ for the 8-electron clusters and $R \approx 10.7 \AA$ for the 138 -electron cluster.

Table 1: The parameters of the studied dimer systems. $S$ is the separation between the jellium edges and $a$ is the radius of the cylindrical linking channel in the case of linked dimers. $r \approx 4.2 \AA$ and $R \approx 10.7 \AA$ refer to the radii of the 8-electron and 138-electron clusters, respectively. See figure 1 a) for a visual guide to the parameters.

\begin{tabular}{|l|l|l|l|}
\hline system & number of el. & $S$ & $a(\AA)$ \\
\hline $\mathbf{1}$ & $8+8$ & $2 r$ & - \\
$\mathbf{2}$ & $8+8$ & $0.5 r$ & - \\
$\mathbf{3}$ & $8+8$ & 0 & - \\
$\mathbf{4}$ & $8+8$ & $2 r$ & 1.0 \\
$\mathbf{5}$ & $8+8$ & $0.5 r$ & 1.0 \\
$\mathbf{6}$ & $8+8$ & $0.5 r$ & 2.1 \\
$\mathbf{7}$ & $138+138$ & $0.5 R$ & - \\
$\mathbf{8}$ & $138+138$ & $0.25 R$ & - \\
$\mathbf{9}$ & $138+138$ & $0.1 R$ & - \\
$\mathbf{1 0}$ & $138+138$ & $0.5 R$ & 1.0 \\
$\mathbf{1 1}$ & $138+138+2$ & $0.5 R$ & 2.1 \\
$\mathbf{1 2}$ & $8+138$ & $0.5 R$ & - \\
\hline
\end{tabular}

Dimers of 8-electron clusters. The absorption spectra and the CTR spectra for the 8-electron cluster dimers can be seen in figure 2. The spectra are for light polarized in the direction of the dimer axis. The CTR values are calculated at the energies of the electronic excitations seen in the oscillation strength spectra without broadening. The upper row is for separate clusters and the lower for linked.

For dimer 1 with a separation of $S / r=2$, there is virtually no electron-cloud overlap, as can be seen from figure S1 a). The absorption spectrum is almost identical to that of a single cluster. ${ }^{41}$ Here, the CTR is also very close to zero along the whole spectrum, indicating that there is no charge transfer between the clusters at any energies.

When the clusters are brought closer together, however, the increasing overlap causes 

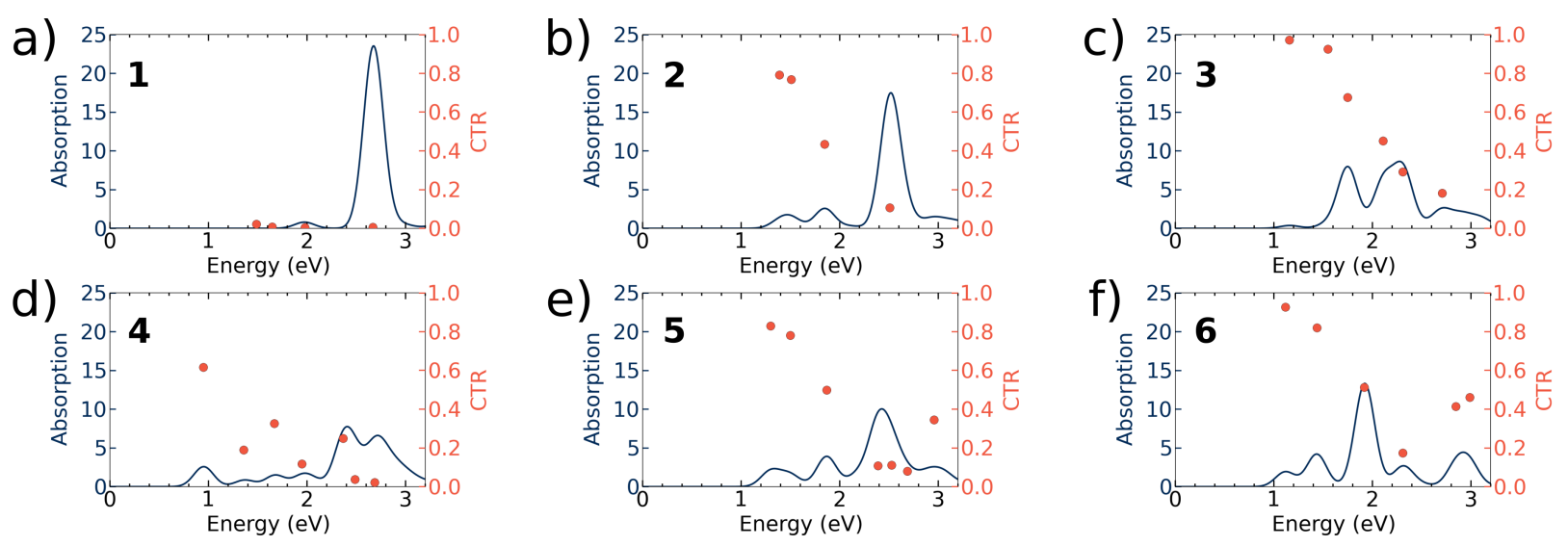

Figure 2: The absorption spectrum and the CTR for the dimers $\mathbf{1 - 6}$ with two 8-electron clusters.

coupling: although the BDP peak is still clearly the strongest peak for dimer $\mathbf{2}$ with the separation-radius ratio $S / r=0.5$, some of the strength has already been transferred to lower energies. In the absorption spectrum this is seen as a red shift of the main peak and the appearance of an extra peak at energies less than $2 \mathrm{eV}$. The lowest-energy excitations have some charge-transfer nature, as proved by the CTR analysis discussed later. For dimer 3 with touching cluster, $S / r=0$, the shape of the spectrum is already very different from separate cluster, with two larger peaks, and a significant CTR value even near $2 \mathrm{eV}$.

Going from larger to smaller separation, we can see the transfer of oscillation strength from the BDP peak to lower energies and excitations of CT nature. The spectrum is very sensitive to even small changes of a couple of Angströms in the separation. This can be explained by changes in the energies and shapes of the electron orbitals, and by the drastic changes in the electron density between the clusters, as seen in figures S1 a) -b).

We can also couple the clusters by connecting them with a conducting linker, as for dimers 4-6. From figures $\mathrm{S} 1 \mathrm{~d}$ )-e) we can see that some of the electron density is now located in the linker. For the systems with the narrower linker $(1.0 \AA), \mathbf{4}$ and $\mathbf{5}$, the electron density between the clusters is only moderately bigger than for the separate clusters. For dimer 6 with the stronger linker, however, the electron density in the linker is comparable to that of inside the clusters, allowing the electrons to flow freely between the two clusters. 

same inter-cluster distance, dimer 1. For dimer 4 there is not anymore one main peak, and there are many new peaks at energies lower from the BDP peak. All the peaks below $2 \mathrm{eV}$ have non-zero CTR values.

Bringing the clusters closer together, to $S / r=0.5$, but keeping the linker radius constant, the spectrum becomes again more compressed, and less peaks can be seen in the spectrum of dimer 5. However, the CTR is higher for the smallest peaks for the smaller separation. If we, conversely, keep the distance constant $S / r=0.5$ but increase the linker width to $2.1 \AA$, the change in the positions of the clearest peaks is not very drastic, but the relative strengths of the peaks change, as is evident from the spectrum of dimer 6 .

For the systems 3-6 with a strong coupling, the BDP peak is fragmented. Also some of these fragments have some charge transfer nature, but the CTR value goes to minimum in this energy range, indicating that the electron density oscillations happen mostly separately inside each cluster.

We will now study in more detail one absorption peak with a large CTR value for three of the systems. In figure 3 are shown the DTCMs and the induced densities for one prominent CTP peak in systems $\mathbf{2}, \mathbf{3}$, and $\mathbf{4}$ each. In all of the systems, the induced densities of the strongest CTP resonances have a similar shape when looked at an appropriate isosurface value. Here, the isosurface value is the same for each system, which makes the weaker plasmon for system $\mathbf{2}$ appear to have a different shape.

The main contributions to the dipole moment come from $\mathrm{P} \rightarrow \mathrm{D}$ transitions. The coupling between the two clusters has caused some splitting in the orbitals for each system, the most in system 3 with the touching clusters. This hybridization of orbitals due to coupling is an absolute requirement for charge transfer excitations. The first 20 orbitals (1S, 1P, 1D, and $2 \mathrm{~S}$ symmetries) for dimers $\mathbf{2 - 4}$ are visualized in figures $\mathrm{S} 1-\mathrm{S} 4$. For systems $\mathbf{2}$ and $\mathbf{3}$, the lowest-energy $1 \mathrm{P}$ orbital is the bonding state of the individual $\mathrm{P}$ orbitals, aligned in the 
a)



2 at $1.51 \mathrm{eV}$


b)



3 at $1.75 \mathrm{eV}$

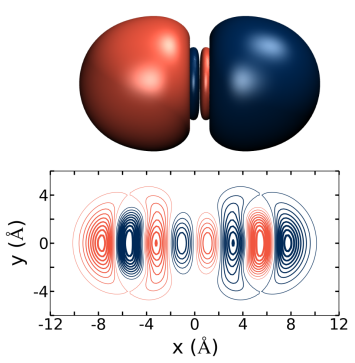

c)

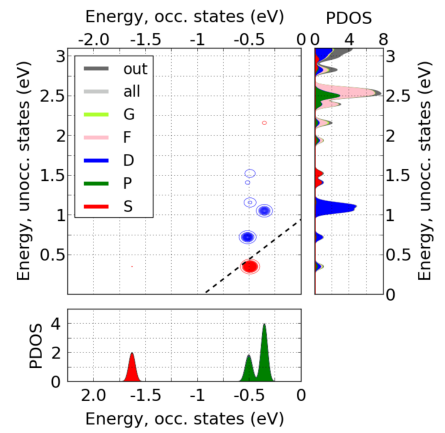

4 at $0.95 \mathrm{eV}$
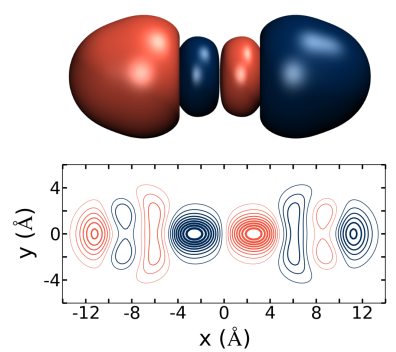

Figure 3: The DTCMs and induced densities for the $2 \cdot 8$ electron dimers $\mathbf{2}, \mathbf{3}$, and $\mathbf{4}$ for one CTP peak at energies indicated in the figure. The contour plot shows the relative strength of the contributions of each e-h transition to the induced dipole moment, read indicating positive and blue negative contribution. The colors of the projected density of states in the DTCM figures indicate the symmetries of the orbitals, as projected for spherical harmonics. The symmetry analysis is done for both clusters separately. The dashed lines are for $E_{e x}=\epsilon_{\text {unocc }}-\epsilon_{o c c}$, where $E_{e x}$ is the energy of the excitation, $\epsilon_{\text {unocc }}$ is the energy of the unoccupied K-S state, and $\epsilon_{\text {occ }}$ is the energy of the occupied K-S state. The induced densities are visualized as isosurfaces with a certain postive and negative value of the same absolute value in the middle row. The bottom row shows a contour plot of the induced density at $z=0$, along the dimer plane. The isosurface values are the same for each system. 

antibonding $\left(\pi^{*}\right)$ states of orbitals aligned in different directions, and finally HOMO is the antibonding state of the individual $\mathrm{P}$ orbitals aligned along the dimer axis $\left(\sigma^{*}\right)$.

For the two separate dimers $\mathbf{2}$ and $\mathbf{3}$, the HOMO-LUMO transition is one of the strongest positive contributions. LUMO in both systems is a bonding state of D orbitals, but for $\mathbf{2}$ of the $\mathrm{D}_{z}^{2}$ orbitals, and for $\mathbf{3}$ of the other type of $\mathrm{D}$ orbitals. For $\mathbf{3}$, another positive contribution is from $\mathrm{P}$ symmetry $\pi^{*}$ to LUMO. For $\mathrm{B}$, the other positive contribution is $\mathrm{P}$ symmetry $\pi^{*}$ to a bonding state of $\mathrm{D}$ orbitals. For $\mathbf{3}$, there is a contribution from $\pi^{*}$ to LUMO and from HOMO to the antibonding state of $2 \mathrm{~S}$ orbitals. Both systems have at least one screening contribution from $\pi$ to antibonding states of 1D.

For the linked dimer, system 4 , the order of the 1P type orbitals is different: from lower to higher energy, the states are $\sigma, \sigma^{*}, 2 \times \pi$ and $2 \times \pi^{*}$, so the orbitals with more electron density in the linker are lower in energy. Of these, the $\sigma$ and $\sigma^{*}$ orbitals are very close to each other in energy, and the $\pi$ and $\pi^{*}$ orbitals are almost degenerate. Here, the positive contribution comes from transition from the $\mathrm{P}$ symmetry $\sigma^{*}$ orbital to the bonding state of the $\mathrm{D}_{z}^{2}$ orbitals aligned along the dimer axis. The main negative contributions are from $\mathrm{P}$ type $\sigma$ to the antibonding state of $\mathrm{D}_{z}^{2}$ orbitals aligned along the dimer axis and from the different $\sigma$ or $\sigma^{*}$ orbitals to different $\mathrm{D}$ orbital states.

Dimers of 138-electron clusters. The absorption spectra and CTR spectra for the 138-electron cluster dimers can be seen in figure 4. In the upper row are again separate clusters, from larger separation to smaller, and in the bottom row two linked systems with the same separation but different linker width. For these larger clusters, the density of states is bigger than for the 8-electron clusters, and thus the spectrum is more continuous, leading to clearer trends in the response of the spectrum to changes in geometry. Going to smaller separations, more and more absorption strength is transferred to smaller energies, which correspond to excitations with a large CTR. The CTR value has a minimum near the BDP peak. Linking the clusters has a similar effect, except the BDP peak is better preserved. 

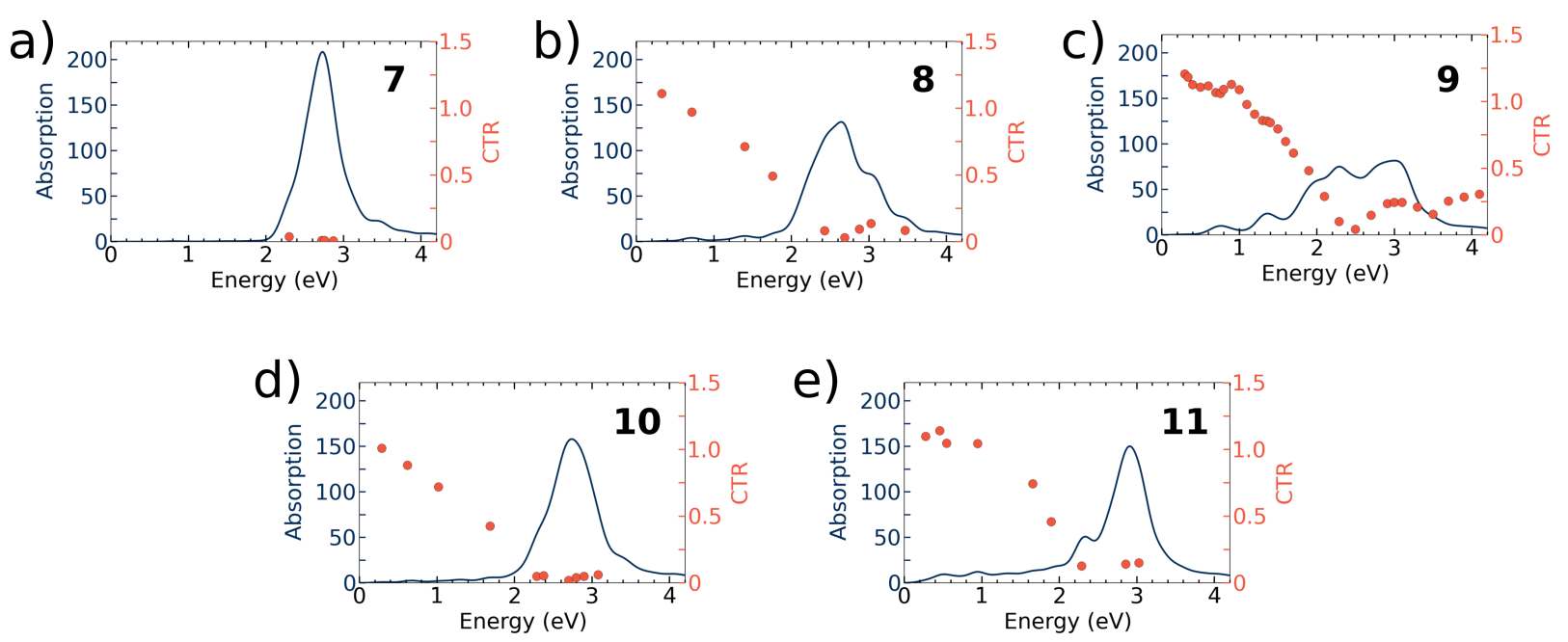

Figure 4: The absorption spectrum and the CTR for the dimers $\mathbf{7 - 1 1}$ with two 138-electron clusters.

One should notice that since the linker widths are the same here as they were for the smaller clusters, the linkers for these larger systems are relatively narrower compared to the cluster size.

The energies of the BDP and CTP peaks agree approximately with those obtained by Rossi et al. ${ }^{29}$ using TDDFT for dimeric sodium systems with a total of 261 atoms. They varied the linking between the clusters in the dimer by starting from an uniform nanorod and stretching it to first form a narrow metallic bridge between two thicker ends and then finally breaking the system to two parts. The dimers with a narrow linker were found to have two CTP peaks between 0 and $1 \mathrm{eV}$ and one BDP peak between 2 and $2.5 \mathrm{eV}$. The completely separate clusters supported a second BDP peak between 1 and $1.5 \mathrm{eV}$. Here, the highest CTR values are for absorption peaks below $1.5 \mathrm{eV}$, and the BDP peak, although strongly fragmented for systems $\mathbf{8}$ and $\mathbf{9}$, is found between 2 and $3 \mathrm{eV}$. Our results are also qualitatively similar to experimental studies of dimeric silver ${ }^{20}$ and gold nanoparticles ${ }^{19,21}$ and covalently linked gold clusters, ${ }^{22}$ with a CTP peak appearing at energies smaller than the BDP peak for small separations or touching or linked clusters.

If $2 \cdot 138$ electrons are placed in the potential of the positive jellium background with the wider linker (2.1 , the Wigner-Seitz radius used for the jellium background), the dimer 
a)

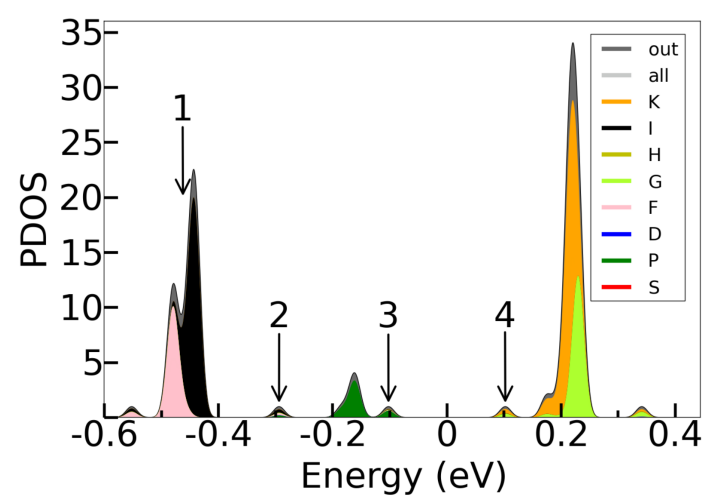

b)
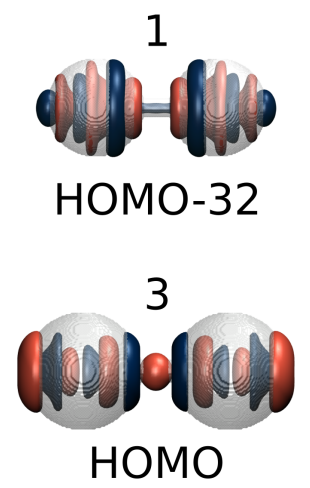
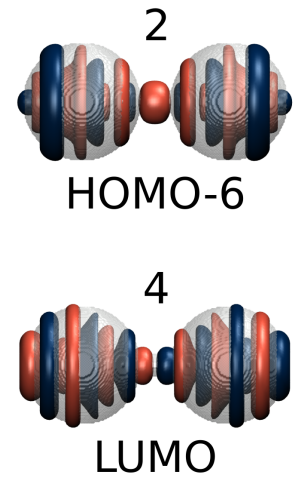

Figure 5: a) The projected density of states near the HOMO-LUMO gap and b) selected orbitals for dimer 11. The arrows and numbers in a) indicate the orbitals that are shown in b). The jellium surface of the dimer is shown in transparent.

is not anymore a closed-shell system, with the HOMO-LUMO gap in the middle of the 3P states. This is caused by an extra electron orbital, HOMO-32, forming between the $2 \mathrm{G}$ and 1I orbitals. The orbital has some G and I symmetry inside the two clusters separately, but it is clearly asymmetric inside one cluster, and symmetric only with respect to the whole system. The positive and negative isosurfaces of this orbital can be seen in figure $5 \mathrm{~b}$ ), where it is orbital number 1 . To keep all the studied systems closed-shell, we then added two electrons and calculated the properties of this system. The dimer with the wider linker studied here has thus $2 \cdot 138+2$ electrons, two electrons more than the rest of the bigger dimers. The order and symmetries of the electronic states stayed the same after the addition of these extra electrons.

Closer inspection of the PDOS and the orbitals show that the orbitals aligned in the direction of the dimer axis are markedly separated in energy from the other orbitals, causing splitting in the DOS - the energy of the bonding combination of these orbitals is lowered, and the energy of the antibonding combination raised. The shapes of these orbitals are also distorted from the combination of the orbitals of two non-interacting clusters, making them quite asymmetric inside one cluster, and only symmetric regarding the whole dimer. Three of these orbitals are visualized in figure 5 , where they are labeled as $2-4$. In total, there is a 
considerable amount of electron density in the linker, as can be seen from figure S1 k). As a result, the electrons can freely flow between the two clusters.

a)

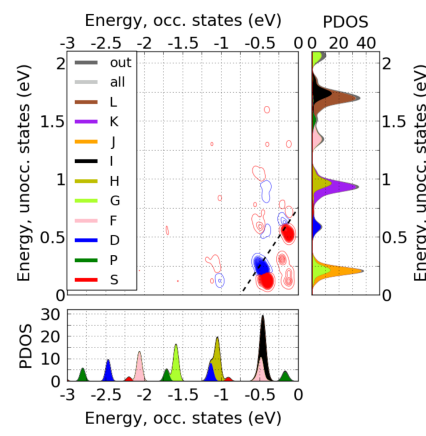

9 at $0.76 \mathrm{eV}$

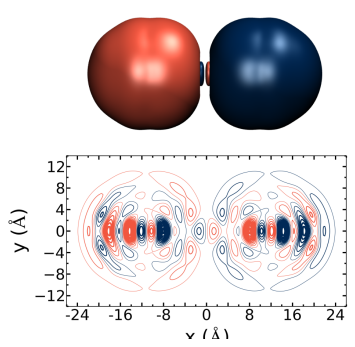

b)

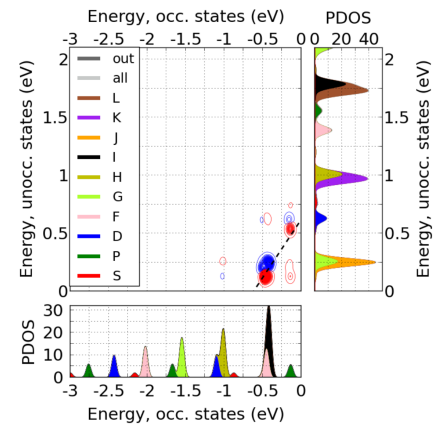

10 at $0.62 \mathrm{eV}$
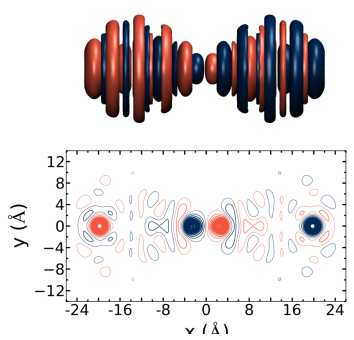

C)

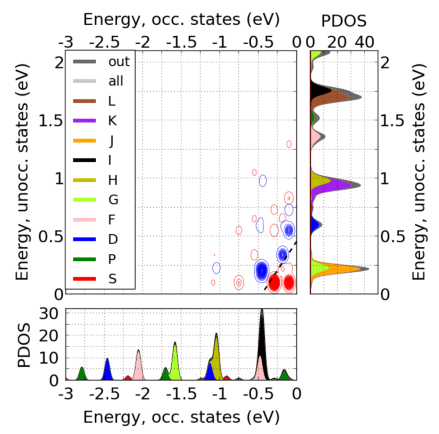

11 at $0.46 \mathrm{eV}$

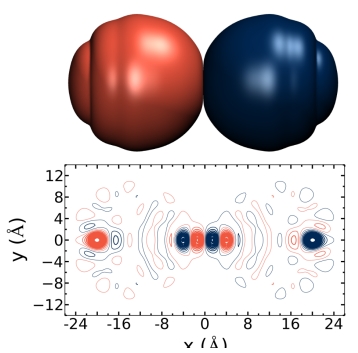

Figure 6: The DTCMs and induced densities for the $2 \cdot 138$ electron dimer systems 9, 10, and $\mathbf{1 1}$ for one CTP peak at energies indicated in the figure. See the caption of figure 3 for details.

The DTCMs and the induced densities for one peak with a high CTR value are shown for systems 9, $\mathbf{1 0}$ and $\mathbf{1 1}$ in figure 6. In the DTCM, the difference between CTP and $\mathrm{BDP}$ excitations is that in the former there are stronger contributions from the screening transitions. The transitions are still mainly those that are allowed for a single spherical cluster, except of the transitions of the HOMO orbitals to the LUMO in system 11. These orbitals are the ones aligned along the dimer axis and having significant electron density in the linker.

As the CTR values in figure 4 show, for all of the energies analyzed in figure 6 , in total one of the clusters is negatively and the other positively charged. However, there are also induced density variations inside the clusters. For the separate clusters, in system 9, the induced density inside each cluster still somewhat resembles the induced density for the 
LSPR peak for a single cluster ${ }^{41}$ reflecting the shell structure of the electron density, which can be seen in figures S1 g)-i). Here, the highest density changes are near the center of the clusters. For the linked clusters, the picture changes. For system 10, with the narrower linker, the biggest values for the induced density along the dimer axis are found at both ends of the clusters, forming two temporary dipoles inside each cluster. For the dimer with the wider linker, system 11, additionally an extra dipole is formed in the linker.

From the DTCMs in figure 5 we can see that for systems $\mathbf{9}$ and 10, the contributions come from transitions from $1 \mathrm{I}$ to $1 \mathrm{~K}, 2 \mathrm{~F}$ to $2 \mathrm{G}$, and $3 \mathrm{P}$ to $3 \mathrm{D}$. The main positive contributions for system 11 are from transitions from the HOMO-6 and HOMO orbitals to LUMO, as visualized in figure 5. The strongest negative contribution comes from transitions from the $1 \mathrm{I}$ orbitals to $1 \mathrm{~J}$ orbitals, although there are some clearly weaker contributions from $3 \mathrm{P}$ to $3 \mathrm{D}$ and $3 \mathrm{P}$ to $1 \mathrm{~J} / 2 \mathrm{G}$ transitions.
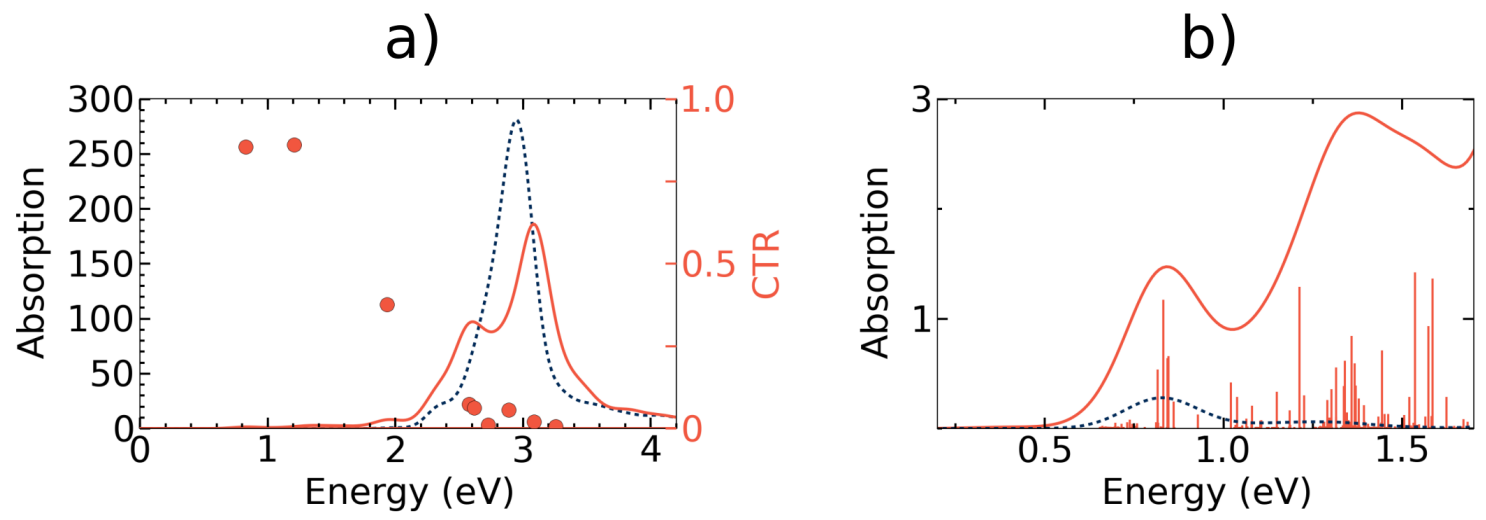

Figure 7: a) The absorption spectrum of the dimer 12 with 8- and 138-electron clusters (solid orange line) and the summed absorption spectra of the separate clusters with 8 and 138 electrons (dashed blue line). The CTR values are for the dimer. b) A zoom of the spectra between $0.2 \mathrm{eV}$ and $1.8 \mathrm{eV}$, along with the oscillation strengths for the dimer.

Heterodimer. The heterodimer (dimer 12) has two clusters of different sizes: the 138electron cluster with a radius of $10.7 \AA$ and the 8-electron cluster with a radius of $4.2 \AA$. The separation between the jellium edges was $2.7 \AA$, which is $1 / 4$ of the radius of the larger cluster. The electron density overlap between the clusters, as shown in figure S1 1), is similar to that of the dimer of the 138-electron clusters with the same separation, seen in S1 h). In 
figure $7 \mathrm{a}$ ), the spectrum of the heterodimer is plotted in orange and the sum of the spectra of the two separate clusters in blue. The CTR values are calculated for the dimer. The sides $\mathrm{A}$ and $\mathrm{B}$ of the calculation box for equation 1 are again chosen so that the division goes halfway between the jellium edges of the cluster. In the ground state, this division gives a total of eight electrons on the side of the smaller cluster, and 138 on the side of the larger cluster when integrating over the electron density. Figure 7 b) shows a zoom of the region between 0.2 and $1.8 \mathrm{eV}$, with the oscillation strengths for the heterodimer visible. In figure 8 are shown the DTCMs and induced densities for the heterodimer at three energies.

The individual 138-electron cluster has its LSPR peak at $2.95 \mathrm{eV}$, and the 8-electron cluster at $2.72 \mathrm{eV} .{ }^{41}$ For the heterodimer, the two largest peaks for light polarized in the dimer axis direction are found at $2.61 \mathrm{eV}$ and $3.08 \mathrm{eV}$. The CTR value is close to zero for both of these peaks, indicating little or no charge transfer between the cluster. Also the other analysed excitations at energies $2.6 \mathrm{eV}-3.1 \mathrm{eV}$ have small CTR values.

For the first of the largest peaks, that is analyzed in figure $8 \mathrm{~b}$ ), the induced density inside the smaller cluster is similar to that of the individual 8-electron cluster at the LSPR energy. From the contour plot we can see that most of the induced density is concentrated around the smaller cluster. The main transition of the isolated smaller cluster, $1 \mathrm{P} \rightarrow 1 \mathrm{D}$, is contributing to the dipole moment also in this system, but many transitions inside the larger cluster are participating in the excitation too.

The shape of the induced density distribution for the largest peak is similar to the induced density of the LSPR peak for the individual 138-electron cluster. Also the main contributions to the dipole moment come from the same e-h transitions as in the single cluster. However, there are also changes in density inside the smaller cluster, and the $1 \mathrm{P} \rightarrow 1 \mathrm{D}$ transitions have some screening contribution. Here the density oscillations inside the bigger cluster are almost symmetric, as can be seen from the contour plot of the induced density, but very asymmetric inside the smaller cluster.

The latter peak around $3 \mathrm{eV}$ is clearly smaller in the heterodimer than the LSPR peak 
a)
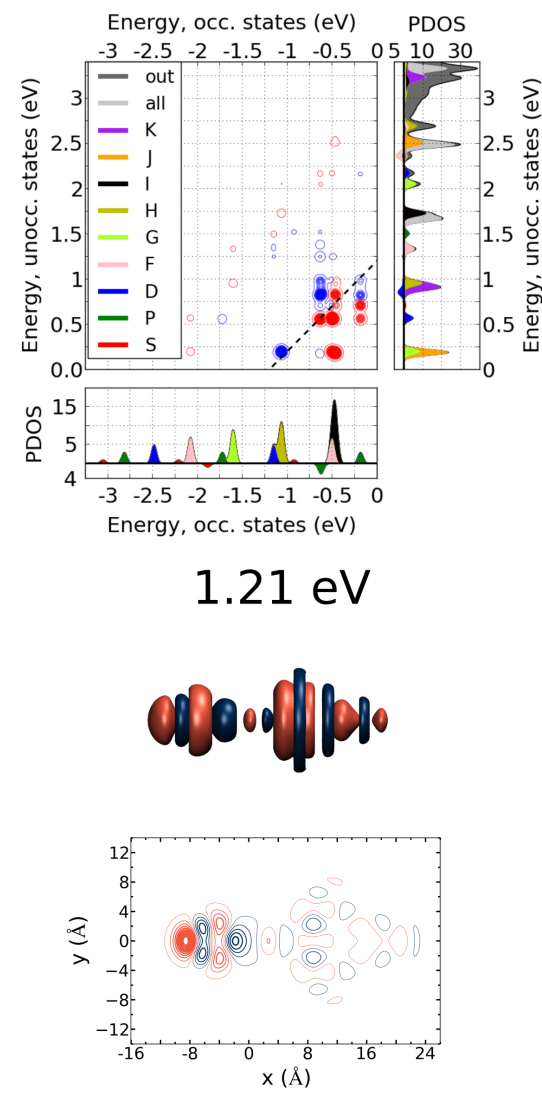

b)
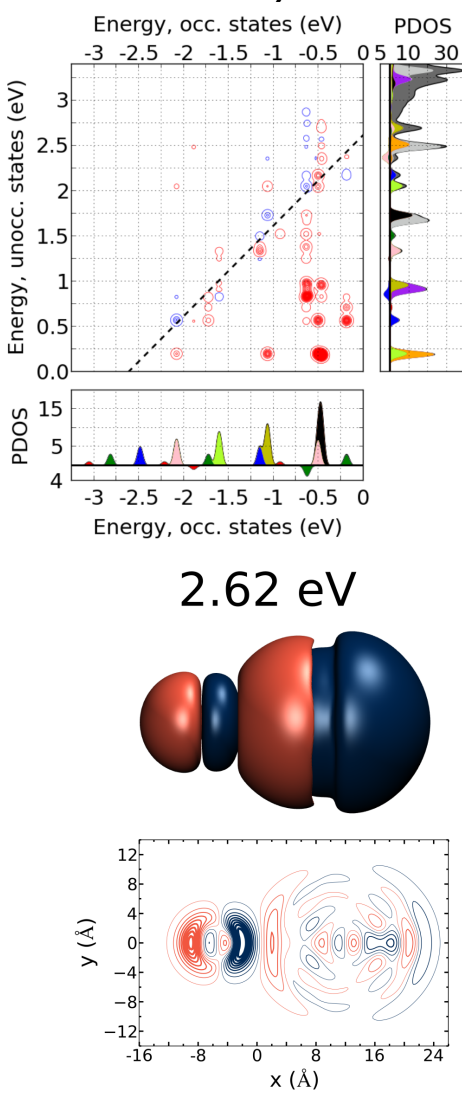

C)
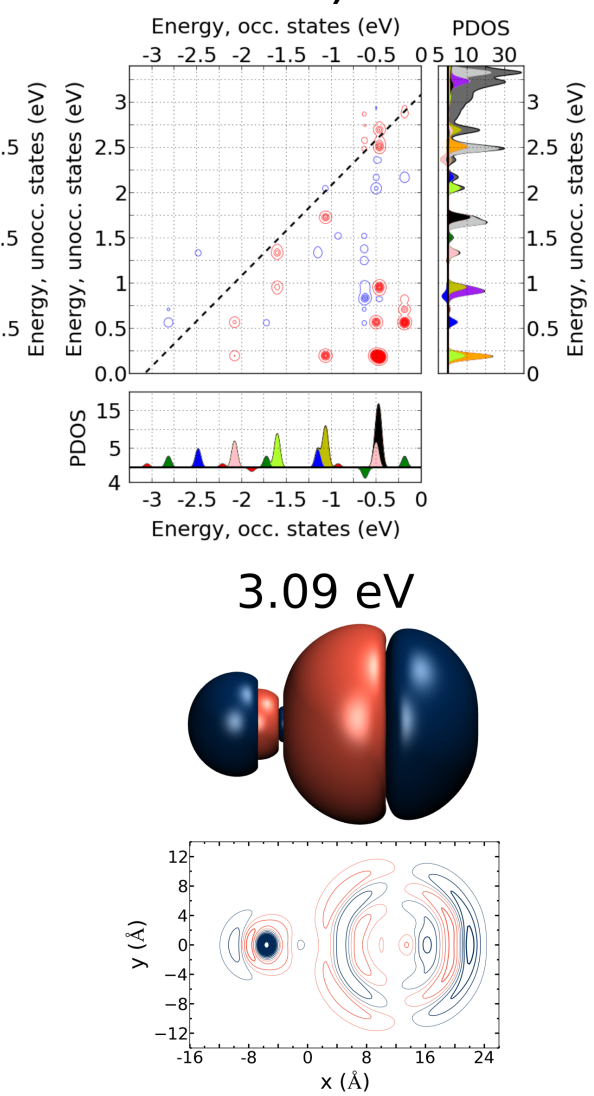

Figure 8: The DTCMs and induced densities for three different peaks for the heterodimer at energies indicated in the figure. Here, the PDOS analysis is plotted separately for the smaller cluster (down) and for the larger cluster (up). See further details in the caption of figure 3 . 
for the individual 138-electron cluster, and the peak at $2.62 \mathrm{eV}$ is larger than the LSPR peak for the 8-electron cluster. A clear coupling is thus present between the clusters, and the larger cluster 'borrows' some oscillation strength to the smaller one. Since the shape, the energy of the largest peak, and the maximum absorption strength of the spectrum is changing drastically from the single 138-electron cluster to the heterodimer, the larger cluster can also be thought of as a sensor, as the presence of the smaller cluster can be detected from the optical response of the system. The amplification of the main peak for the smaller cluster also helps in this detection.

The CTR values are larger for the excitations below $2 \mathrm{eV}$ in energy. One of these excitations, at $1.21 \mathrm{eV}$, is analyzed in figure $8 \mathrm{a}$ ). At this energy, there are no excitations in the absorption spectra of the individual clusters, as can be seen from figure $7 \mathrm{~b}$ ). For this excitation, the biggest changes in the electron density are around the smaller cluster. The induced density distribution is also quite asymmetric inside both of the clusters. The difference in the DTCM compared to the higher energy peaks is the presence of many screening transitions (blue color), one of which is the $1 \mathrm{P} \rightarrow 1 \mathrm{D}$ transition of the smaller cluster.

\section{Conclusions}

We have studied the coupling of plasmons and the emergence of the charge transfer plasmon in dimers of metal clusters from the electronic perspective using the simple jellium model. Here all the systems had cylindrical symmetry, but the real-space grid based GPAW code enables the study of clusters and cluster assemblies of any kind of geometries. The high scalability of the code made possible the lr-TDDFT calculations of the systems with the larger dimers of 276 or 278 electrons, where the number of electron-hole pairs included in the calculation was approximately 90000 .

The Charge Transfer Ratio index, calculated post-processing from the induced densities, makes it possible to indentify the excitations with the most charge transfer nature. The CTR 
spectra show that the excitations visible at small energies in the absorption spectra of dimers with electron cloud overlap or linking all have charge transfer nature. These transitions are not present in the spectrum of dimers with a large separation,

For the smaller clusters, the effect of the linker on the optical spectra is quite drastic. Here, the radius of the narrower linker is about $1 / 4$, and the wider linker $1 / 2$ of the radius of the spheres, which changes the electron density profile and the energies of the orbitals significantly from that of separate clusters with the same separation. For the larger clusters, the ratio of the radius of the linker and the radius of the sphere is about 0.20 for the wider linker. For these dimers, the shape of the spectra changes less with linking, but especially for the wider linker, a significant amount of the oscillation strength is transferred to low-energy charge transfer nature excitations. The wider linker, the radius of which corresponds to the Wigner-Seitz radius used for the jellium density, also enables the emergence of a new occupied K-S orbital.

One heterodimer system with an 8-electron and 138-electron cluster was also studied. The larger cluster is observed to borrow oscillation strength to the smaller, so that the LSPR peak of the larger cluster is diminished while the LSPR peak for the smaller cluster gains strength. Some excitations with charge transfer nature are also present at lower energies.

Experimental systems such as cluster dimers linked with ligands, colloidal cluster assemblies and arrays of clusters on a substrate all require knowledge of the effect of the coupling of the electronic excitations to the optical properties. The jellium linker used here can act as a simplified model for one or several conducting linker molecules, or metal atom chain between clusters, showing the trend for the absorption spectrum when conductive linker is introduced. When modeling the complex ligand-protected clusters, the results of these simple jellium clusters can help to sort the different kind of excitations and to understand the underlying principles of the electronic behavior. 


\section{Author information}

\section{Corresponding Author}

Hannu Häkkinen, Departments of Physics and Chemistry, Nanoscience Center, University of Jyväskylä, FI-40014 Jyväskylä, Finland; Email: hannu.j.hakkinen@jyu.fi

\section{Authors}

Elli Selenius, Department of Physics, Nanoscience Center, University of Jyväskylä, FI40014 Jyväskylä, Finland

Sami Malola, Department of Physics, Nanoscience Center, University of Jyväskylä, FI-40014 Jyväskylä, Finland

Mikael Kuisma, Department of Chemistry, Nanoscience Center, University of Jyväskylä, FI-40014 Jyväskylä, Finland

\section{Notes}

The authors declare no competing financial interest.

\section{Acknowledgement}

This work was supported by the Academy of Finland (grants 294217 and 319208, H.H.'s Academy Professorship, and M.K.'s Academy postdoctoral grant 295602) and the Emil Aaltonen Foundation (E.S.'s PhD scholarship). The computations were done at the CSC - the Finnish IT Center for Science (project COUPLES) and in the FGCI - Finnish Grid and Cloud Infrastructure (persistent identifier urn:nbn:fi:research-infras-2016072533).

\section{Supporting Information Available}

- The electron densities along the dimer axis for all the dimers, the 20 first electron orbitals for dimers 2-4, a discussion about the choice of the LDA functional, and derivation of the CTR equation. This information is available free of charge at ... 


\section{References}

(1) Kelly, K. L.; Coronado, E.; Zhao, L. L.; Schatz, G. C. The Optical Properties of Metal Nanoparticles: The Influence of Size, Shape, and Dielectric Environment. J. Phys. Chem. B 2003, 10\%, 668-677.

(2) Su, K.-H.; Wei, Q.-H.; Zhang, X.; Mock, J.; Smith, D. R.; Schultz, S. Interparticle Coupling Effects on Plasmon Resonances of Nanogold Particles. Nano Lett. 2003, 3, $1087-1090$.

(3) Nie, S.; Emory, S. R. Probing Single Molecules and Single Nanoparticles by SurfaceEnhanced Raman Scattering. Science 1997, 275, 1102-1106.

(4) Sönnichsen, C.; Reinhard, B. M.; Liphardt, J.; Alivisatos, A. P. A Molecular Ruler Based on Plasmon Coupling of Single Gold and Silver Nanoparticles. Nat. Biotechnol. 2005, 23, 741-745.

(5) Hirsch, L. R.; Stafford, R. J.; Bankson, J. A.; Sershen, S. R.; Rivera, B.; Price, R.; Hazle, J. D.; Halas, N. J.; West, J. L. Nanoshell-Mediated Near-Infrared Thermal Therapy of Tumors Under Magnetic Resonance Guidance. Proc. Natl. Acad. Sci. U.S.A. 2003, 100, 13549-13554.

(6) Linic, S.; Aslam, U.; Boerigter, C.; Morabito, M. Photochemical Transformations on Plasmonic Metal Nanoparticles. Nat. Mater. 2015, 14, 567-576.

(7) Guidez, E. B.; Aikens, C. M. Quantum Mechanical Origin of the Plasmon: from Molecular Systems to Nanoparticles. Nanoscale 2014, 6, 11512-11527.

(8) Li, J.-H.; Hayashi, M.; Guo, G.-Y. Plasmonic Excitations in Quantum-Sized Sodium Nanoparticles Studied by Time-Dependent Density Functional Calculations. Phys. Rev. $B$ 2013, 88, 155437. 
(9) Townsend, E.; Bryant, G. W. Which Resonances in Small Metallic Nanoparticles Are Plasmonic? J. Opt. 2014, 16, 114022.

(10) Bernadotte, S.; Evers, F.; Jacob, C. R. Plasmons in Molecules. J. Phys. Chem. C 2013, 117, 1863-1878.

(11) Casanova, D.; Matxain, J. M.; Ugalde, J. M. Plasmonic Resonances in the $\mathrm{Al}_{13}$-Cluster: Quantification and Origin of Exciton Collectivity. J. Phys. Chem. C 2016, 120, 1274212750.

(12) Bursi, L.; Calzolari, A.; Corni, S.; Molinari, E. Quantifying the Plasmonic Character of Optical Excitations in Nanostructures. ACS Photonics 2016, 3, 520-525.

(13) Zhang, R.; Bursi, L.; Cox, J. D.; Cui, Y.; Krauter, C. M.; Alabastri, A.; Manjavacas, A.; Calzolari, A.; Corni, S.; Molinari, E. et al. How to Identify Plasmons from the Optical Response of Nanostructures. ACS Nano 2017, 11, 7321-7335.

(14) Gieseking, R. L.; Ashwell, A. P.; Ratner, M. A.; Schatz, G. C. Analytical Approaches to Identify Plasmon-Like Excited States in Bare and Ligand-Protected Metal Nanoclusters. J. Phys. Chem. C 2020, 124.

(15) Nordlander, P.; Oubre, C.; Prodan, E.; Li, K.; Stockman, M. Plasmon Hybridization in Nanoparticle Dimers. Nano Lett. 2004, 4, 899-903.

(16) Lassiter, J. B.; Aizpurua, J.; Hernandez, L. I.; Brandl, D. W.; Romero, I.; Lal, S.; Hafner, J. H.; Nordlander, P.; Halas, N. J. Close Encounters Between Two Nanoshells. Nano Lett. 2008, 8, 1212-1218.

(17) Zuloaga, J.; Prodan, E.; Nordlander, P. Quantum Description of the Plasmon Resonances of a Nanoparticle Dimer. Nano Lett. 2009, 9, 887-891. 
(18) Savage, K. J.; Hawkeye, M. M.; Esteban, R.; Borisov, A. G.; Aizpurua, J.; Baumberg, J. J. Revealing the Quantum Regime in Tunnelling Plasmonics. Nature 2012, $491,574-577$.

(19) Fontana, J.; Charipar, N.; Flom, S. R.; Naciri, J.; Piqué, A.; Ratna, B. R. Rise of the Charge Transfer Plasmon: Programmable Concatenation of Conductively Linked Gold Nanorod Dimers. ACS Photonics 2016, 3, 904-911.

(20) Scholl, J. A.; García-Etxarri, A.; Koh, A. L.; Dionne, J. A. Observation of Quantum Tunneling Between Two Plasmonic Nanoparticles. Nano Lett. 2013, 13, 564-569.

(21) Su, M.-N.; Sun, Q.; Ueno, K.; Chang, W.-S.; Misawa, H.; Link, S. Optical Characterization of Gold Nanoblock Dimers: from Capacitive Coupling to Charge Transfer Plasmons and Rod Modes. J. Phys. Chem. C 2018, 122, 18005-18011.

(22) Lahtinen, T.; Hulkko, E.; Sokołowska, K.; Tero, T.-R.; Saarnio, V.; Lindgren, J.; Pettersson, M.; Häkkinen, H.; Lehtovaara, L. Covalently Linked Multimers of Gold Nanoclusters $\mathrm{Au}_{102}(p-\mathrm{MBA})_{44}$ and $\mathrm{Au}_{250}(p-\mathrm{MBA})_{n}$. Nanoscale 2016, 8, 18665-18674.

(23) Jain, P. K.; Huang, W.; El-Sayed, M. A. On the Universal Scaling Behavior of the Distance Decay of Plasmon Coupling in Metal Nanoparticle Pairs: a Plasmon Ruler Equation. Nano Lett. 2007, 7, 2080-2088.

(24) Varas, A.; García-González, P.; Feist, J.; García-Vidal, F.; Rubio, A. Quantum Plasmonics: from Jellium Models to Ab Initio Calculations. Nanophotonics 2016, 5, 409426.

(25) Zhu, W.; Esteban, R.; Borisov, A. G.; Baumberg, J. J.; Nordlander, P.; Lezec, H. J.; Aizpurua, J.; Crozier, K. B. Quantum Mechanical Effects in Plasmonic Structures with Subnanometre Gaps. Nat. Commun. 2016, \%, 1-14. 
(26) Zhao, L. L.; Jensen, L.; Schatz, G. C. Surface-Enhanced Raman Scattering of Pyrazine at the Junction between Two $\mathrm{Ag}_{20}$ Nanoclusters. Nano Lett. 2006, 6, 1229-1234.

(27) Zhao, K.; Troparevsky, M. C.; Xiao, D.; Eguiluz, A. G.; Zhang, Z. Electronic Coupling and Optimal Gap Size Between Two Metal Nanoparticles. Phys. Rev. Lett. 2009, 102, 186804.

(28) Bae, G.-T.; Aikens, C. M. TDDFT and CIS Studies of Optical Properties of Dimers of Silver Tetrahedra. J. Phys. Chem. A 2012, 116, 8260-8269.

(29) Rossi, T. P.; Zugarramurdi, A.; Puska, M. J.; Nieminen, R. M. Quantized Evolution of the Plasmonic Response in a Stretched Nanorod. Phys. Rev. Lett. 2015, 115, 236804.

(30) Mokkath, J. H. Nanoparticle Heterodimers: The Role of Size and Interparticle Gap Distance on the Optical Response. Chem. Phys. Lett. 2018, 699, 28-31.

(31) Alkan, F.; Aikens, C. M. Understanding Plasmon Coupling in Nanoparticle Dimers Using Molecular Orbitals and Configuration Interaction. Phys. Chem. Chem. Phys 2019, 21, $23065-23075$.

(32) Xiang, H.; Zhang, M.; Zhang, X.; Lu, G. Understanding Quantum Plasmonics from Time-Dependent Orbital-Free Density Functional Theory. J. Phys. Chem. C 2016, 120, 14330-14336.

(33) Esteban, R.; Borisov, A. G.; Nordlander, P.; Aizpurua, J. Bridging Quantum and Classical Plasmonics with a Quantum-Corrected Model. Nat. Commun. 2012, 3, 19.

(34) Esteban, R.; Zugarramurdi, A.; Zhang, P.; Nordlander, P.; García-Vidal, F. J.; Borisov, A. G.; Aizpurua, J. A Classical Treatment of Optical Tunneling in Plasmonic Gaps: Extending the Quantum Corrected Model to Practical Situations. Faraday Discuss. 2015, 178, 151-183. 
(35) Song, P.; Nordlander, P.; Gao, S. Quantum Mechanical Study of the Coupling of Plasmon Excitations to Atomic-Scale Electron Transport. J. Chem. Phys. 2011, 134, 074701.

(36) Song, P.; Meng, S.; Nordlander, P.; Gao, S. Quantum Plasmonics: SymmetryDependent Plasmon-Molecule Coupling and Quantized Photoconductances. Phys. Rev. $B$ 2012, 86, 121410 .

(37) Marinica, D. C.; Kazansky, A. K.; Nordlander, P.; Aizpurua, J.; Borisov, A. G. Quantum Plasmonics: Nonlinear Effects in the Field Enhancement of a Plasmonic Nanoparticle Dimer. Nano Lett. 2012, 12, 1333-1339.

(38) Aguirregabiria, G.; Marinica, D. C.; Esteban, R.; Kazansky, A. K.; Aizpurua, J.; Borisov, A. G. Role of Electron Tunneling in the Nonlinear Response of Plasmonic Nanogaps. Phys. Rev. B 2018, 97, 115430.

(39) Zhang, P.; Feist, J.; Rubio, A.; García-González, P.; García-Vidal, F. Ab Initio Nanoplasmonics: The Impact of Atomic Structure. Phys. Rev. B 2014, 90, 161407.

(40) Barbry, M.; Koval, P.; Marchesin, F.; Esteban, R.; Borisov, A. G.; Aizpurua, J.; Sánchez-Portal, D. Atomistic Near-Field Nanoplasmonics: Reaching Atomic-Scale Resolution in Nanooptics. Nano Lett. 2015, 15, 3410-3419.

(41) Selenius, E.; Malola, S.; Häkkinen, H. Analysis of Localized Surface Plasmon Resonances in Spherical Jellium Clusters and Their Assemblies. J. Phys. Chem. C 2017, 121, 27036-27052.

(42) Mortensen, J. J.; Hansen, L. B.; Jacobsen, K. W. Real-Space Grid Implementation of the Projector Augmented Wave Method. Phys. Rev. B 2005, 71, 035109.

(43) Enkovaara, J.; Rostgaard, C.; Mortensen, J. J.; Chen, J.; Dułak, M.; Ferrighi, L.; Gavnholt, J.; Glinsvad, C.; Haikola, V.; Hansen, H. A. et al. Electronic Structure 
Calculations with GPAW: a Real-Space Implementation of the Projector AugmentedWave Method. J. Phys. Condens. Matter 2010, 22, 253202.

(44) Perdew, J. P.; Wang, Y. Accurate and Simple Analytic Representation of the ElectronGas Correlation Energy. Phys. Rev. B 1992, 45, 13244-13249.

(45) Walter, M.; Häkkinen, H.; Lehtovaara, L.; Puska, M.; Enkovaara, J.; Rostgaard, C.; Mortensen, J. J. Time-Dependent Density-Functional Theory in the Projector Augmented-Wave Method. J. Chem. Phys. 2008, 128, 244101.

(46) Andrade, X.; Botti, S.; Marques, M. A.; Rubio, A. Time-Dependent Density Functional Theory Scheme for Efficient Calculations of Dynamic (Hyper) Polarizabilities. J. Chem. Phys. 2007, 126, 184106.

(47) Malola, S.; Lehtovaara, L.; Enkovaara, J.; Häkkinen, H. Birth of the Localized Surface Plasmon Resonance in Monolayer-Protected Gold Nanoclusters. ACS Nano 2013, 7, 10263-10270.

(48) Rossi, T. P.; Kuisma, M.; Puska, M. J.; Nieminen, R. M.; Erhart, P. Kohn-Sham Decomposition in Real-Time Time-Dependent Density-Functional Theory: an Efficient Tool for Analyzing Plasmonic Excitations. J. Chem. Theory Comput. 2017, 13, 47794790.

(49) Walter, M.; Akola, J.; Lopez-Acevedo, O.; Jadzinsky, P. D.; Calero, G.; Ackerson, C. J.; Whetten, R. L.; Grönbeck, H.; Häkkinen, H. A Unified View of Ligand-protected Gold Clusters as Superatom Complexes. Proc. Natl. Acad. Sci. U.S.A. 2008, 105, 9157-9162. 

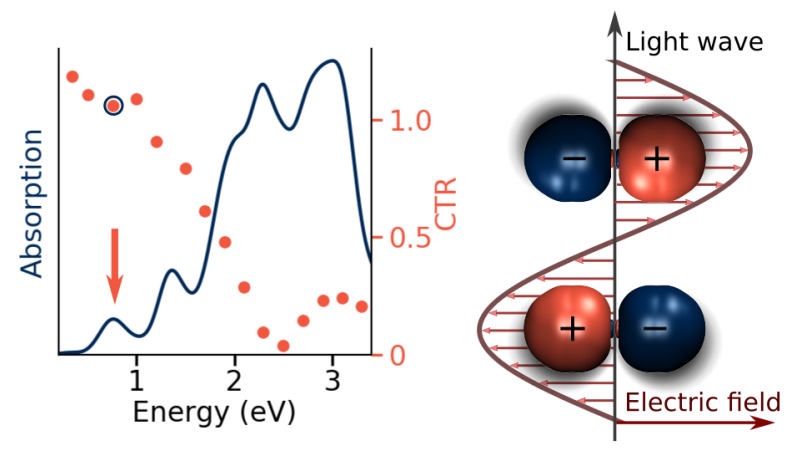

TOC 